\title{
Effects of Low \& High Literacy on User Performance in Information Search and Retrieval
}

\author{
Neesha Kodagoda \\ Interaction Design Centre \\ School of Computing Science, \\ Middlesex University, London, UK \\ +44 (0)7931800 0357 \\ neesha.kodagoda@gmail.com
}

\author{
B L William Wong \\ Interaction Design Centre \\ School of Computing Science, \\ Middlesex University, London, UK \\ +44 (0)20 84112684 \\ w.wong@mdx.ac.uk
}

\begin{abstract}
This study was part of research into understanding the nature of how low literacy users search for and retrieve information, and to therefore develop systems and user interface designs that would empower low literacy users to find information they need in the rapidly evolving e-government and e-social services environment. We compared information search and retrieval performance between high and low literacy users of a Citizens Advice Bureau information kiosk system in the UK. The kiosk provided self-help information in a number of social services areas. Six high literacy and six low literacy users were presented with information search tasks classified as having low, medium and high complexity. Key results indicate that (i) low literacy users take eight times more time than high literacy users to complete an information search task, and yet were significantly less accurate, (ii) low literacy users on average spent one-third more time on a web page than high literacy users, but did not seem to be informed by it, (iii) low literacy users employed a much less focused information search strategy than high literacy users visiting eight times more web pages in total, (iv) low literacy users back-tracked 13 times more frequently than high literacy users, and are four times more likely to re-visit web pages, and (v) low literacy users are 13 times more likely to be lost than high literacy users.
\end{abstract}

\section{Keywords}

Low and high literacy, Information search task, Information search, Information retrieval

\section{INTRODUCTION}

As information technologies expand far beyond the traditional personal computer into public information systems, the designers of these systems should consider the target audience and their level of literacy.

Barnet Citizens Advice Bureau, or CAB, is a local charity providing free, impartial, confidential advice and information to

\section{(C) The Author 2008 .}

Published by the British Computer Society people who live, work or study in the London Borough of Barnet regardless of race, gender or ability they provide information and advice on benefits, tax credits, consumer goods services, debt, education, employment, financial product services, health, community care, housing, immigration asylum and nationality issues, legal aid, relationship and family advice, signposting and referral, travel transport and holidays, utilities and communications, and on other related issues to its citizens.

A primary objective of public service authorities is to provide information to its citizens. It is also crucial to optimise the quality of such services by considering usage patterns and user characteristics, for example, their age, vocational status (student, employed, unemployed, unskilled, retired), literacy level and disability. These determine user clusters or groups. The needs of one group, such as recent refugee migrants who need advice on mental health information will be far different from another group like unemployed youth requiring advice regarding mental health support (Wong, Keith, \& Springett, 2006). This would vary according to group, literacy and sociocultural expectations and suggests that different information delivery methods for diverse user groups are required. The problem is further complicated by dependencies between group problems. For example, an unemployed youth could come with mental health issues, poor skills, low income, debt and a criminal record - the information is scattered across several government departments (silos) such as websites of the Department of Health, the Department of Work and Pension and council services making retrieval of relevant information more difficult (Wong, Keith, \& Springett, 2006).

The main focus in this research is on persons with low levels of literacy defined according to National Skills for Life Survey. Low level of literacy could be due to English not being their first language, or leaving school at an early (and an inappropriate) stage. These factors may result in subsequent poor ability to write or to spell adequately to meet the demands of daily life.

This paper discusses some of the key findings from a series of evaluations on how the level of literacy among the users influenced the behaviour of information search and retrieval, and future work. 12 users of the Barnet $\mathrm{CAB}$ participated in the study. They were screened for literacy, and were asked to carry out a series of information search and retrieval tasks, based on a number of frequently reported queries by clients of the CAB. The next section reports on some background before we report on the study. 


\section{RELATED WORK}

Literacy is the ability to read, write and count in one's primary language, to take advantage of the information sources available and to compose short meaningful statements (Baynham, 1995, Chlebowaka, 1990, Stifelman, et. al 1993). Literacy screening has been carried out since World War I, with the Army Alpha and Beta test applied to about 1.9 million men (William, et al. 2003) . This was to assess the mental ability of adults who were eligible for military service. The Army Alpha intelligence test was to evaluate literate participants while the Beta intelligence test was focused on illiterates or non-English speaking participants. The results help to assign jobs suited to participants' abilities (William, et al. 2003).

Low literacy users have problems in searching for information, and this is increased by the considerable amount of concentration and effort required to read. They tend to read every word, trying not to miss any out (Chadwick-Dias, et al., 2003, Theofanos, \& Redish, 2003, Theofanos, \& Redish, 2004, Tullis \& Chadwick-Dias, 2003).

The low literacy user have problems attending to detail and difficulty determining what is expected next or where they arrived from, as simply processing the text places high demands on cognitive attention. Due to this, such groups of users have a narrow field of view and might not notice content above, below or to the sides of their focus of attention. Content in adjacent paragraphs should be as independent as possible, making it easier for the user to carry on without having to remember content from the previous paragraphs, to reduce the possibility of getting misinformation (Summers, \& Summers, 2005). Low literacy users may skip chunks of text when presented with long, dense pages, pages that require scrolling, text containing numbers, words that are long, difficult and unfamiliar, or pages containing parenthetical text (Summers, \& Summers, 2005). The low literacy user tends to assume they have enough information and abandons reading further, resulting in incomplete or inaccurate information. Further, low literacy users find searching for information difficult, as it requires proper spelling and typing capabilities (Summers, \& Summers, 2005). The above references to indicate that behavior of low literacy people are not clearly understood under different context.

\section{Definition of Literacy Levels}

The literacy screening method used in our study was the National Skills for Life Survey. The survey tests various aspects of reading, writing, listening, prose comprehension and quantitative skills, along with several grammar and spelling questions. It comprised 40 questions ( 8 listening, 16 reading and 16 writing) spread across different literacy levels. The survey divides literacy into Low Literacy comprising Entry Level 1, 2 and 3; and High Literacy comprising Level 1,2 and above (William, et al. 2003). These literacy levels are defined next.

Entry Level 1 (EL1) - Understands short texts with repeated language patterns on familiar topics and has the ability to obtain information from common signs and symbols. The reading level compares with that of a six year old or younger, at National Curriculum Level 1.

Entry Level 2 (EL2) - Understands short straightforward texts on familiar topics and has the ability to obtain information from short documents, familiar sources and signs and symbols. The reading level expected is that of a seven-year-old, at National Curriculum Level 2.
Entry levels 3 (EL3) - Understands short straightforward text on familiar topics accurately, independently and have the ability to obtain information from everyday sources. The reading level is that expected of an 11-year-old, at National Curriculum Levels $3-4$.

Level 1 (L1) - Understands short straightforward texts of varying length on a variety of topics accurately and independently and have the ability to obtain information from different sources. The reading level is that expected of GCSE grades D-G, at National Curriculum Level 5.

Level 2 or above (L2) - Understands a range of texts of varying complexity accurately and independently and have the ability to obtain information of varying length and detail from different sources. The reading level is that expected of GCSE grades A-C, at National Curriculum Levels 6-8.

\section{Information Seeking and Task Complexity}

Research has been carried out on information seeking behaviours over the last couple of decades. Information seeking is a step where the resolution of a particular task begins. 'Personal Construct' concept of Kelly (1963) was adopted by Kuhlthau (1991). Kuhlthau asserts that information seeking starts with considerable uncertainty. The model consists of stages of initiation, selection, exploration, formulation, collection and presentation. The information seeking model at each stage provides the user affective, cognitive and physical experience. Aguilar (1967), Weick \& Daft (1983) identifies four information seeking models undirected viewing, conditioned viewing, informal search, and formal search. These information seeking models recognise the nature of the task is more focused on a specific goal. A search originates from a task (Belkin, 1980) and the characteristics of the task have an impact on how the user approaches and retrieves information, as well as the difficulty of the task. The task characteristics have an impact on selecting a search strategy.

Byström \& Järvelin (1995) discuss how the task difficulty has a direct effect on the quality of information sought. Campbell (1988) describes task difficulty in subjective three steps involving how the person interacts with the task and the characteristics of the task 1. Psychological (subjective), 2. Person-task interaction and 3. Objective (defined by the task characteristics). He explains that there are four factors that identify the task difficulty; 1 . Having multiple paths to reach its goal, each of which yielding its own outcomes and thus contributing differently to the end goal, 2. Conflicting interdependencies among these paths in reaching the end goal, 3. Doubts whether a given path would materialize into the end goal, and 4. Multiple possible goals. More research has been carried out on assessing the relationship between the task difficulty and information seeking. They go to say that current task difficulty is due to person task interaction (Kim, Y. M., \& Rieh, S. Y. 2005).

\section{METHOD}

One scenario and six information search tasks were developed from the information and advice sought by clients of the Barnet Citizens Advice Bureau. The scenario was based on the most frequently client requested sets of information during the period April 2005 to May 2007.

The scenario describes a young family and their two young children who have recently moved to Barnet. The family tries to find a number of items of information, including a children's 
hospital for the sick toddler, child care information for the older child, information on arrears on council tax payments, and help for their family debts.

In our study, we carried out an experiment based on a $2 \times 3$ factorial design, assessing the effects of literacy levels (high vs low) against level of difficulty (easy, medium and difficult) in an information search task. 12 participants volunteered for this between subjects study, grouped on either low literacy or high literacy. Five of the participants were females and 7 participants were males with an average age of 42 (age ranges from 38 to 52). None of the selected participants for the experiment have used the touch screen kiosks information system or the Barnet website previously. Six of the participants were highly literate; 2 were L1 and 4 were L2. The remainders were low literate, where 4 were EL1 and 2 were EL2. EL3 participants were unavailable during this study. pages. This requires three to four navigation steps with multiple possible paths to find the solution as described by Campbell (1988) on task difficulty. The medium difficulty information search task targeted both low literacy (EL2 and EL3) and high literacy (L1 and L2) participants and consisted of two tasks, M1 and M2.

Difficult - The information requires more reading from external web pages, has multiple possible paths and solutions as described by Campbell (1988). The difficult information search tasks targeted both low literacy (EL3) and high literacy (L1 and L2) participants and consisted of two tasks, D1 and D2.

\section{PROCEDURE}

The study was conducted at the one of the Citizens Advice Bureau's information centres located at a local community hospital. In preparation for the study, each participant was given an explanation of the goals of the experiment and the

Table 1 - Summary of Experimental Results During an Information Search Task

\begin{tabular}{|c|c|c|c|c|c|c|c|c|c|c|}
\hline $\begin{array}{l}\text { Literacy } \\
\text { Level }\end{array}$ & $\begin{array}{l}\text { Task } \\
\text { Difficulty }\end{array}$ & $\begin{array}{l}\text { Task } \\
\text { Completion } \\
\text { Success }\end{array}$ & $\begin{array}{l}\text { User } \\
\text { Assumed } \\
\text { Successfully } \\
\text { Completed }\end{array}$ & $\begin{array}{l}\text { Total } \\
\text { Pages } \\
\text { Visited } \\
\text { ( Pvisited) }\end{array}$ & $\begin{array}{l}\text { Unique } \\
\text { Number } \\
\text { Pages } \\
\text { (Upages) }\end{array}$ & $\begin{array}{l}\text { Average } \\
\text { Time } \\
\text { Page } \\
\text { (Atime) }\end{array}$ & $\begin{array}{l}\text { Total } \\
\text { Time } \\
\text { Spent in } \\
\text { Seconds } \\
\text { (Tspent) }\end{array}$ & $\begin{array}{l}\text { Back } \\
\text { Button } \\
\text { (Bbutton) }\end{array}$ & $\begin{array}{l}\text { Revisits } \\
\text { ( Revisits) }\end{array}$ & $\begin{array}{l}\text { Lostness } \\
\text { (Lostness) }\end{array}$ \\
\hline \multirow{7}{*}{$\begin{array}{l}\text { Low } \\
\text { Literacy }\end{array}$} & E1 & 2 & 6 & 18.33 & 10.33 & 32.36 & 593.33 & 7.17 & $42 \%$ & 0.83 \\
\hline & E2 & 5 & 6 & 19.50 & 10.50 & 26.50 & 516.67 & 7.67 & $44 \%$ & 0.82 \\
\hline & M1 & 0 & 4 & 65.00 & 28.17 & 16.49 & $1,071.67$ & 24.17 & $55 \%$ & 1.10 \\
\hline & M2 & 1 & 3 & 49.33 & 21.17 & 16.93 & 835.00 & 12.50 & $55 \%$ & 1.04 \\
\hline & D1 & 2 & 2 & 39.33 & 19.50 & 20.83 & 819.17 & 12.33 & $53 \%$ & 0.82 \\
\hline & D2 & 2 & 2 & 45.83 & 19.50 & 18.00 & 825.00 & 15.33 & $59 \%$ & 0.87 \\
\hline & \multicolumn{3}{|c|}{ Average Low Literacy Summary } & 39.56 & 18.19 & 21.85 & 776.81 & 13.19 & $51 \%$ & 0.91 \\
\hline \multirow{7}{*}{$\begin{array}{l}\text { High } \\
\text { Literacy }\end{array}$} & $\mathrm{E} 1$ & 5 & 6 & 2.00 & 2.00 & 12.67 & 25.33 & - & $0 \%$ & - \\
\hline & E2 & 5 & 6 & 2.00 & 2.00 & 20.67 & 41.33 & - & $0 \%$ & - \\
\hline & M1 & 4 & 6 & 3.17 & 3.00 & 25.79 & 81.67 & 0.17 & $4 \%$ & 0.01 \\
\hline & M2 & 4 & 6 & 3.50 & 3.17 & 24.52 & 85.83 & 0.17 & $7 \%$ & 0.04 \\
\hline & D1 & 3 & 6 & 10.67 & 6.00 & 17.03 & 181.67 & 3.67 & $39 \%$ & 0.28 \\
\hline & D2 & 4 & 4 & 5.50 & 4.17 & 16.91 & 93.00 & 1.00 & $21 \%$ & 0.07 \\
\hline & \multicolumn{3}{|c|}{ Average High Literacy Summary } & 4.47 & 3.39 & 19.60 & 84.81 & 0.83 & $12 \%$ & 0.07 \\
\hline
\end{tabular}

\section{The Information Search Tasks}

Each information search task required the participant to find a specific piece of information, such as assistance on benefits and money advice, assistance on giving up smoking, details of child care available locally, information on a children hospitals, advice on council tax arrears. The tasks were of varying task complexity: Easy, Medium and Difficult. At each task difficulty level we asked 2 questions. The task difficulty level are described as:

Easy - The required information is available at the startup menu list of the information kiosk system. It does not require more than two navigation steps to find the solution and requires little reading. The easy information search task targeted both low literacy (EL1, EL2 and EL3) and high literacy (L1 and L2) participants and consisted of two tasks, E1 and E2.

Medium - The required information is not available in the startup menu list, and requires navigation to external web necessary procedure. Each participant was also asked if they would require reading assistance when the scenario and the six information tasks were presented. The participants were also given an explanation about the think aloud protocol that they would be expected to carry out during the information search and retrieval tasks, and that they would be video recorded during the experiment. The participants were presented with a practice task to familiarise themselves with the think aloud procedure. They were also informed of their rights and that the research was approved by the University's Research Ethics Committee.

Each participant was given the scenario and the six information search tasks, The goal of each search task was to find a target web page based on the scenario given. Participants controlled the start and end of each task. The order of information search tasks described to the participants was randomised. After reading each question, a starting web page (information kiosk system home page shown in Figure 1 below) was presented to 
the participant. The information search task ended when the participant found the target page or abandoned the search. Participants were not provided with any directions.

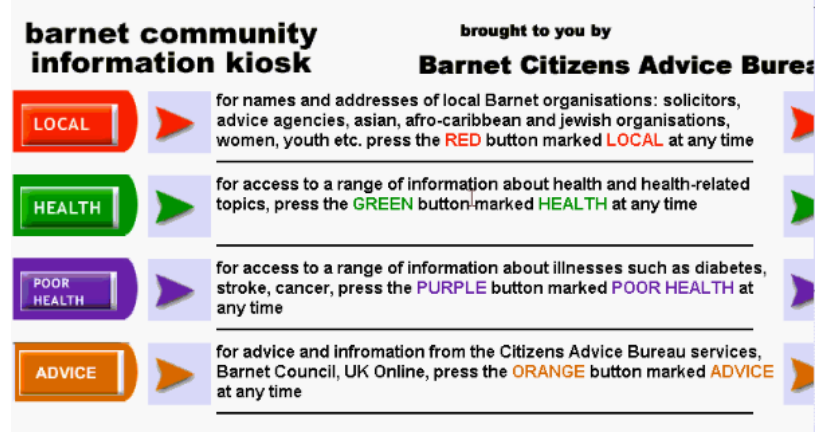

Figure 1 - Screen Shot of the Barnet Community Information Kiosk System

The participants were asked to think aloud during the information search task and the kiosk session was videotaped. After each task was completed, we checked if the information found by the participant was correct. Further, the participants were asked if they believed that they had found what they were looking for (completed, may be completed, not completed) and to rate the task difficulty on a scale of 1 to 5 (easy, medium, difficult, complicated or abandoned).

An individual debriefing interview immediately after the experiment helped to elaborate participants' behaviours during the experiment. Participants watched the recording of their performance and were asked to clarify events and comments on the tape. Retrospection of user actions were identified by questions such as "why did you click on this page," or "why did you keep moving within these pages". Finally, the participants were asked to provide some basic demographic information.

The information kiosks logged time-stamped URLs and video recordings were used to document the participants' behaviour. The measures included the number of total web pages visited during a task (Pvisited), the total time spent during an information task (Tspent), the unique number of pages visited (Upages), number of pages on the optimal path (Opath), the number of times the back button was used (Bbutton) and the average time spent by a searcher on a web page(Atime). These measures are explained in the next section.

\section{RESULTS}

A one-way ANOVA was carried out on the search and retrieval performance data. As the sample size was small $(n=12)$ Levene's test was used to ascertain whether the variances of the two groups, low literacy and high literacy groups, were approximately equal, and hence whether the comparisons between the two groups were valid. If significantly different the data was transformed. If the transformation did not work, the inaccurate $\mathrm{F}$ value was reported along with the effect size for one way independent ANOVA. In this this paper we will not report on the findings from the verbal protocol analysis collected from the think aloud procedure. We next summarise the results of the clients' navigation during the respective information search and retrieval tasks:

Time Taken - This is the average total time taken to complete each information task. Low literacy participants on average spent 760 seconds while the high literacy participants on average spent 85 seconds to solve each information search task (see Table 1 and Figure 2). Statistically significant differences were found in the following tasks M2 F $(1,10)=0.015, \mathrm{p}<0.05$ and $\mathrm{D} 2 \mathrm{~F}(1,10)=3.508, \mathrm{p}<0.05$. The results show that high literacy users were significantly faster than low literacy users in the time taken to complete the information tasks M2 and D2.

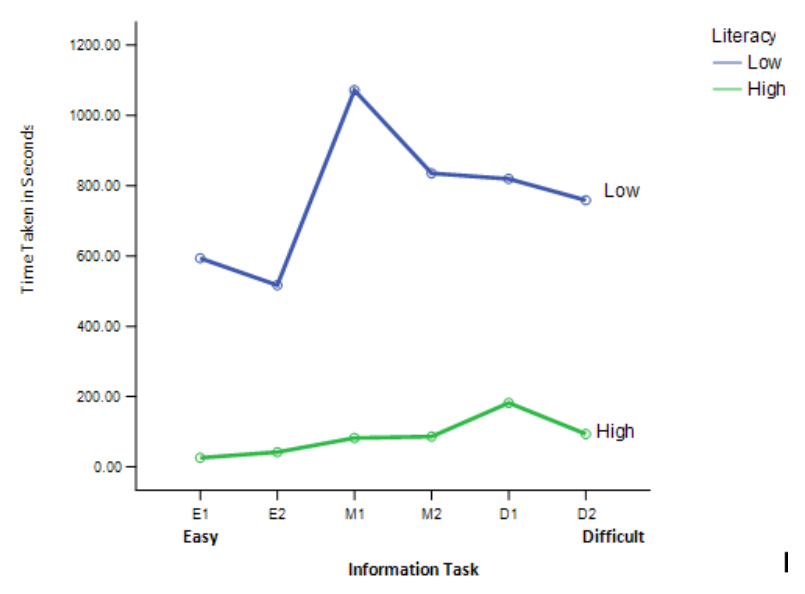

Figure 2 - Taken to Complete Information Search Task

Page Visits - This is the number of pages that a participant visited as they searched for the required information for each information task. Low literacy participants on average viewed 40 web pages per information task, while the high literacy participants on average viewed only 5 pages during each information search task (see Table 1 and Figure 3). Statistically significant differences were found in task D1 F $(1,10)=2.732$, $\mathrm{p}<0.05$. The results show that on information search task D1, low literacy users visited significantly more pages than high literacy users on the same task.

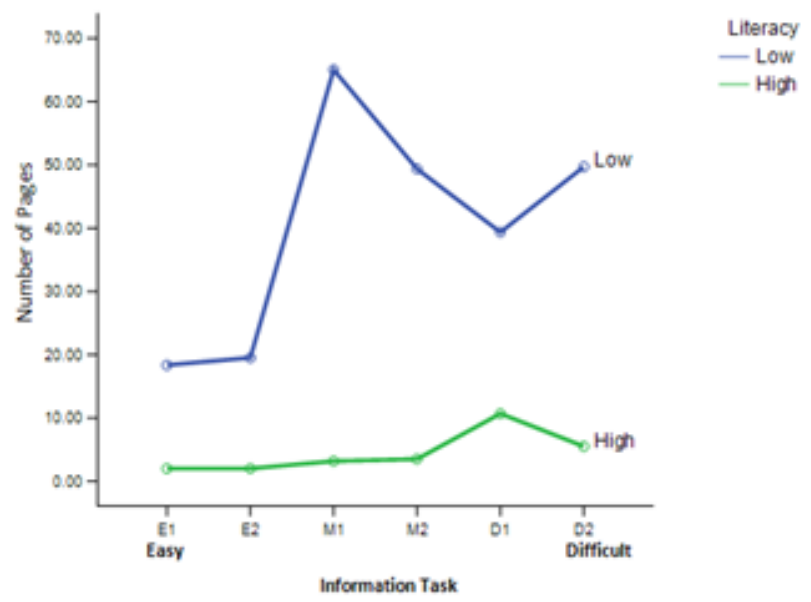

Figure 3 - Total Number of Pages Visited

Back Button Use - This is the number of times that a participant back tracked or returned to a page that was recently visited during a information task. Low literacy participants on average used the back button 13 times and appeared increasingly agitated as the task became more difficult. The high literacy participants used the back button an average of only 0.83 times during each information search task (see Table 1 and Figure 4). Statistically significant differences were found in the task D1 F $(1,10)=1.825, \mathrm{p}<0.05$. For information search 
task D1, the results show that low literacy users used the back button significantly more often than high literacy participants.

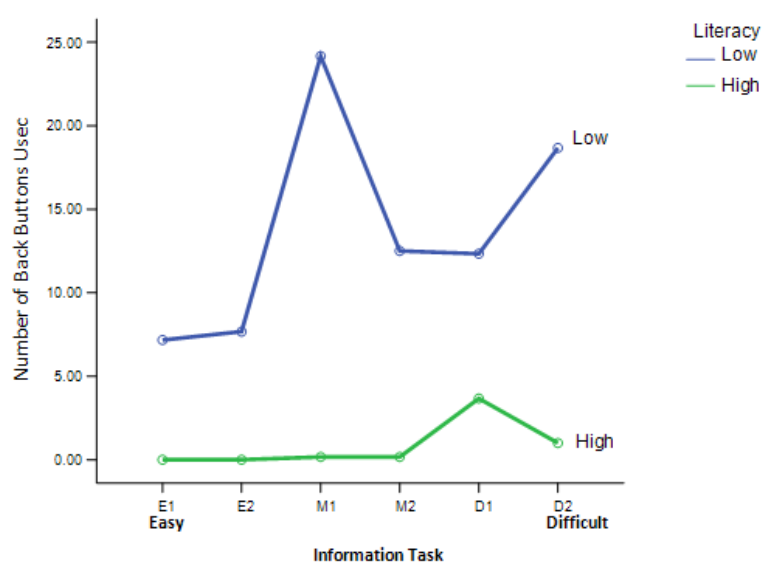

Figure 4 - Back Button Used During an Information Search Task

Unique Page Visits - This is a measure of the number of pages that were visited only once during an information task. Low literacy participants on average visited 18 pages while the high literacy participants on average visited only 4 pages during an information search task. Although the results did not show any statistically the differences, it was still interesting to note that there appeared to be substantial differences in the total number of unique pages visited: Low literacy participants had a much higher count of the number of unique pages visited than the high literacy participants (see Table 1 and Figure 5).

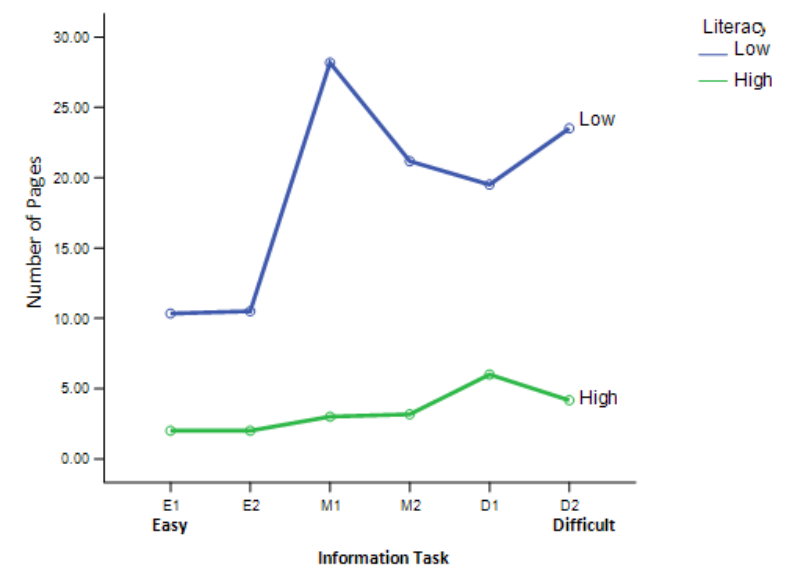

Figure 5 - Unique Number of Pages Visited During an Information Search Task

Time Spent on a Page - This is the measure, in seconds, of the average time that a participant spent between arriving and leaving a web page. Low literacy participants on average spent 24 seconds on a page while the high literacy participants on average spent about 18 seconds on a page during an information search task. Here the low literacy participants appeared or pretended to read the contents, whereas the high literacy participants were observed to browse through the contents.

Revisits - This measure represents the number of times that a participant returned to or re-visited a web page. Revisits was calculated as a percentage of the total number of retrievals carried out on the number of times a user visiting the same web page during an information search task, using the following equation (Catlede, \& Pitkow, 1995):

Revisits $=(($ Pvisited - Upages $) /$ Pvisited $) * 100 \%$

Low literacy participants revisited $51 \%$, of pages and did not seem to recognise they had visited them previously. The average revisit rate for high literacy participants was $12 \%$. Significant differences were found in the following tasks M1 F $(1,10)=0.575, \mathrm{p}<0.05, \mathrm{M} 2 \mathrm{~F}(1,10)=0.009, \mathrm{p}<0.05$, and D1 $\mathrm{F}(1,10)=0.002, \mathrm{p}<0.05$, and $\mathrm{D} 2 \mathrm{~F}(1,10)=0.593, \mathrm{p}<0.05$. The results show that for information search task M1, M2, D1 and D2, the average total number of revisits made by low literacy participants was significantly higher than that made by the high literacy participants (see Table 1 and Figure 6).

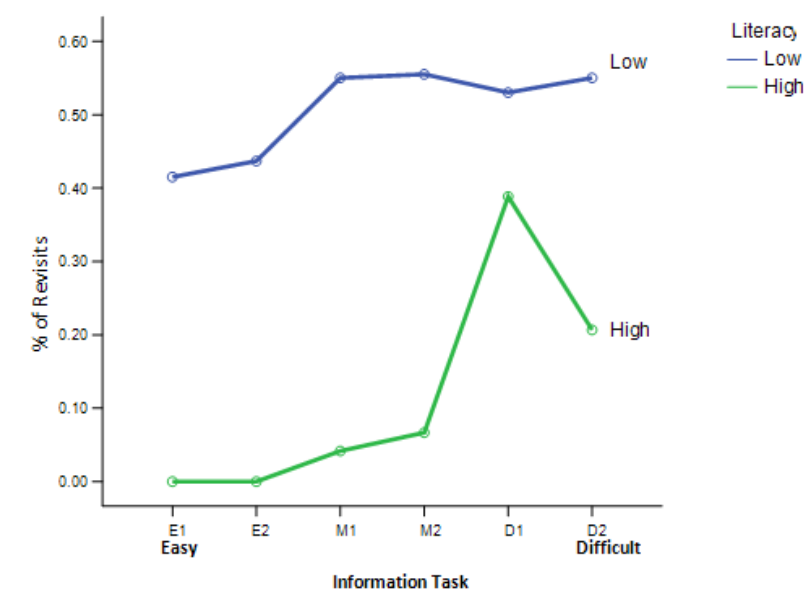

Figure 6 - Revisits During an Information Search Task

Lostness - Lostness is a measure of a participant's disorientation from the task and is calculated using the following equation (Smith, 1996):

Lostness $=\sqrt{ }(\text { Upages } / \text { Pvisited }-1)^{2}+(\text { Opath } / \text { Upages }-1)^{2}$

Upages is the number of unique web pages visited during a search task, Pvisited is the total number of web pages visited during the search and Opath is the required, minimal number of web pages to complete the search task. Lostness is calculated using the ratio of actual web pages visited and the optimal web pages visited during an information search task. Lostness ranges from 0 to 2 .

The lostness of the low literacy participants' was estimated at 0.91 , whereas high literacy participants' lostness was estimated at 0.07 , indicating that low literacy participants seemed to be about 13 times more frequently diverted from the optimal path than are the high literacy users (see Table 1 and Figure 7). 


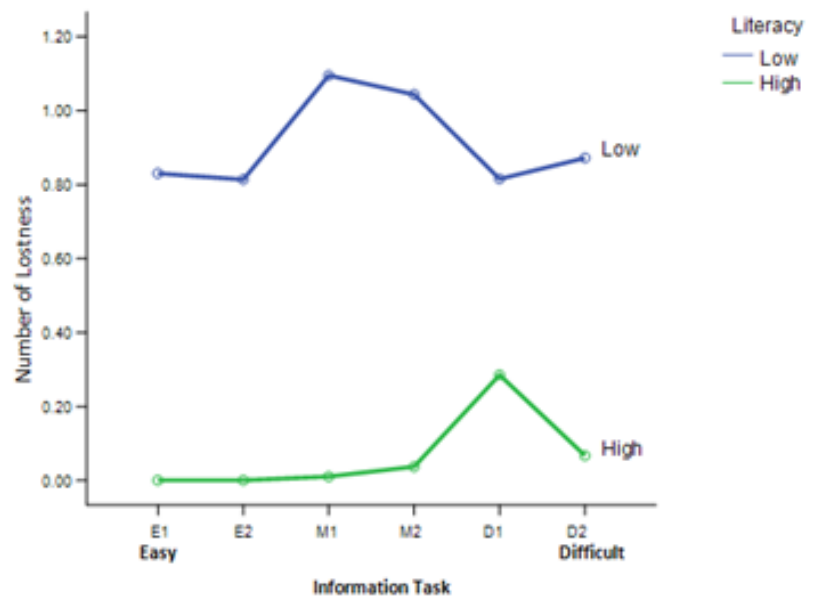

Figure 7 - Lostness Found in Information Search Tasks

Obtained the Goal - The goal for an information task is said to be obtained when the participant correctly declares that he or she has found the required piece of information. This is calculated as a percentage of the correctly declared goals out of the total number of correct goals. Only $17 \%$ of the low literacy participants were able to obtain the goal whereas $61 \%$ of the high literacy participants were able to obtain the goal (see Table 1 and Figure 8).

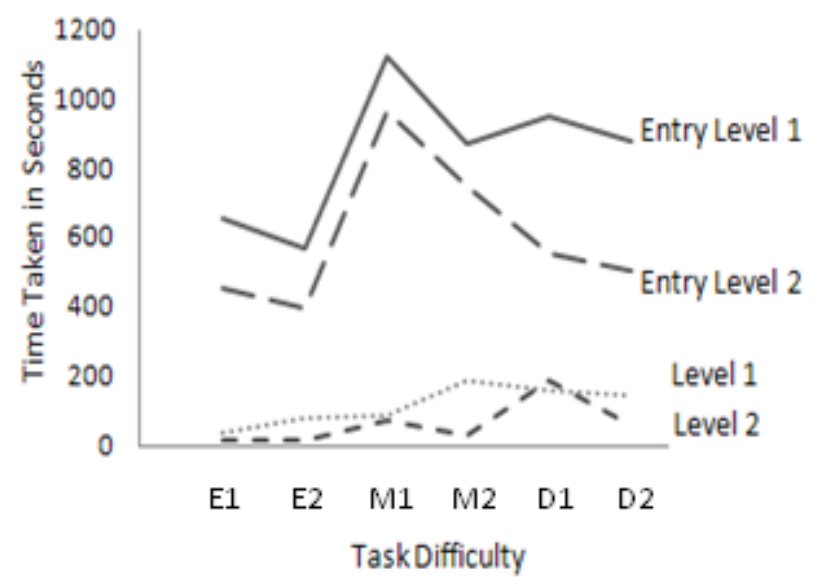

Figure 8 - Mean Time Taken to Complete Task

Assumed obtained the goal - This occurs when a participant incorrectly or prematurely declares that he or she has found the required piece of information, thereby assuming that he or she has obtained they goal, when in fact, has not. $47 \%$ of the low literacy participants abandoned the search early. However, 33\% high literacy participants overlooked details and abandoned the search just before reaching the goal.

Table 2 - A percentage of the Participants Obtained and Assumed they obtained the Goal

\begin{tabular}{ccc}
\hline Literacy Level & $\begin{array}{c}\% \\
\text { Obtained the } \\
\text { Goal }\end{array}$ & $\begin{array}{c}\% \\
\text { Participants Assumed } \\
\text { they Obtained the } \\
\text { Goal }\end{array}$ \\
\hline Low Literacy & $17 \%$ & $64 \%$ \\
High Literacy & $61 \%$ & $94 \%$ \\
\hline
\end{tabular}

Low Literacy participants were 3.8 times more likely to assume they had obtained the goal $(64 \%$ of the participants assumed they obtained the goal, when only $17 \%$ actually did) In comparison, High Literacy participants were only 1.5 times more likely to assume they had obtained the goal $(94 \%$ assumed they did, when only $61 \%$ actually did).

The mean time taken to complete an information search task by the high literacy users (Level 1 \& Level 2) was significantly less than the low literacy users (Entry Level $1 \&$ Entry Level 2) as shown in Figure 7.

Figure 9 shows that significantly more high literacy participants (especially Level 2) achieved their information search task goals, whereas the low literacy participants (Entry Level 1 and Entry Level2) were not been able to achieve their information search task goals.

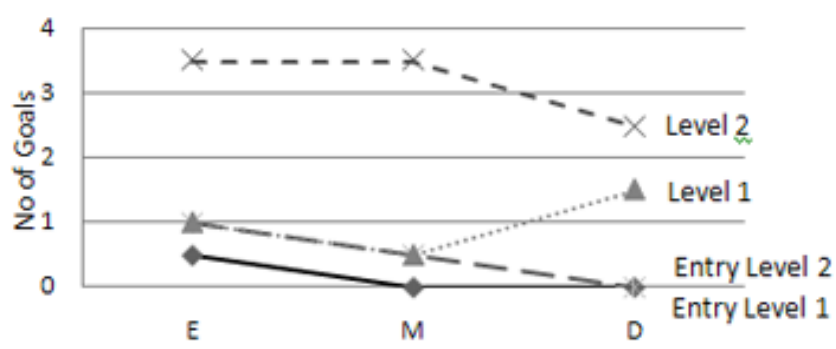

Task Difficulty

Figure 9 - Number of Participants Obtained the Goals

Low literacy participants spent more time during an information search task, visiting a greater number of pages. The number of visited pages directly relates to the number of clicks and to the number of relevance judgments and navigation decisions. This gives an indication of physical and cognitive effort. Low literacy participants higher back button use resulted in high revisits. This indicates they backtracked from their original path and tried new paths. This could be due to change in search strategy.

\section{DISCUSSION}

The participants in our study used the home page of the Barnet Citizens Advice Bureau website as the starting point. Participants were asked to navigate through the site using only the standard navigational mechanisms available in the website, and not to use external search engines. Each information search task led to a specific target piece of information. In this section, we will summarise and discuss our findings from the study in a way that allows us to characterise the information search and retrieval behaviours of the low literacy participants in our study, in relation to the high literacy participants. These findings are summarised next and they suggest that:

(a) Low literacy users take eight times more time than high literacy users to complete an information search task, and yet were significantly less accurate in finding the target information: only $17 \%$ of them correctly found the target information, as compared with $61 \%$ of high literacy users.

(b) Low literacy users were observed to, on average spend onethird more time on a web page than high literacy users, where they appeared to look at the page, but did not seem to be informed by it. 
(c) Low literacy users are more likely to employ a less focused search strategy than high literacy users, visiting eight times more web pages in total than high literacy users in their search for the specific information needed. They also visited four and a half times more 'new' web pages that were not previously visited than high literacy users.

(d) Low literacy users back-tracked more frequently than high literacy users, using the browsers's back button 13 times more frequently than high literacy users, and four times more likely than high literacy users to re-visit web pages. This indicates a high degree of un-successful search paths arising, possibly, from the use of the many more less-than-optimal search paths.

(e) Finally, low literacy users are likely to be 13 times more lost than high literacy users.

The low literacy users on average spent 8 times longer on a search task and visited 8 times more number of pages; as a result of having their navigational path different from the optimal path. The optimal path is the shortest path to solve an information search task. As the task difficulty increases, the average time likely spent on an information search task by low literacy users against the high literacy users reduced respectively; easy 16 times, medium 11 time and difficult to 6 times. Results suggest when task difficulty increases; it affects and increases the low and high literacy users average time spent and number of page visits. In Gwizdka and Spence (2006), higher time spent on an information search task and higher number of pages visit indicated the difficulty of the search task and indicates the effort expanded. The number of page visits relates to the number of clicks, which indicates the relevant navigational judgments and decisions the user requires to make.

A page revisits takes place in two ways (1) when a user backtracks or users the back button (2) by following links on a web page. The low literacy users higher back button use and the larger revisit ratio correspond to users disorientation from the original navigation path and move to new navigational paths. In Gwizdka and Spence (2006) work, discuss higher back tracking and higher revisits ratio results changes in users search strategies that involves higher cognitive effort.

The higher number of unique page visits indicates a less focused search, observed in low literacy users. They were likely to visit 4.5 times more number of unique pages than high literacy user. Lostness, which is the users disorientation from the search task (Smith, 1996) was 13 times more for the low literacy users. The relationship with higher amount of time spent on a search task, higher number of page visits, higher number of unique page visits, higher number of back tracks and high revisits resulted in high lostness user disorientation, less focused searches, and seen in low literacy users.

Our study shows that low literacy users seem to use very different information search strategies than high literacy users. From this, we can hypothesise that low literacy users during an information search task make many navigational judgments that result in much less focused and much less efficient search strategies than do the high literacy users. This could possibly be due to higher mental effort required by them to make sense of the presented information and to therefore employ a more trial and error approach than the high literacy users.

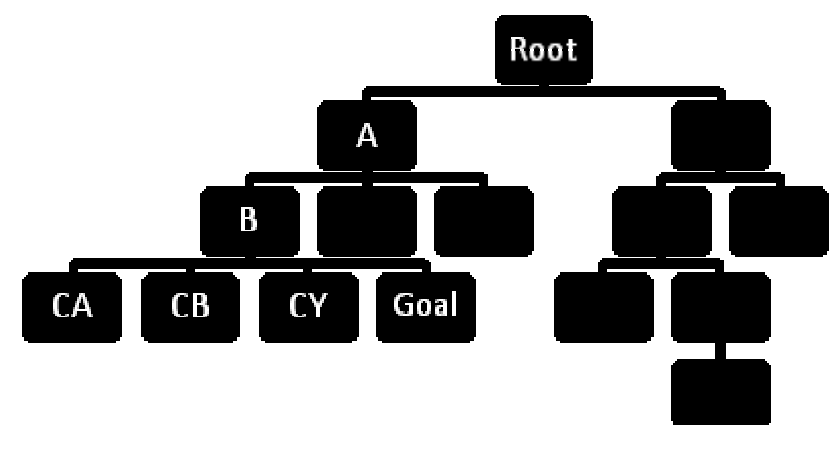

Figure 10 - Information Search Task M1

For example, in one of the medium difficulty information search tasks, M1, illustrated in Figure 10, the participants were asked to "find contact details of child care available locally". Two out of the six low literacy subjects came quite close to the goal via web pages $\mathrm{A}$ and $\mathrm{B}$ and then went through the path $\mathrm{C}$ A to the Goal, and then traversed back and forth revisiting the same pages. They appeared to be confused and frustrated and did not seem to realize they had reached the goal. Both of these low literacy subjects expressed confusion and one of them said, "have I seen this page before? ... hmmm is this what I am to look for? ... where am I? ... how can I get to where I first started?". The remaining four were not in the correct path to reach the goal. They had a high usage of the back button. They did not seem to understand how they had arrived at a certain point and seemed disoriented. Whereas, of the six high literacy participants, three came up to point B and assumed they had reached the goal and abandoned the search early. The remaining three high literacy participants found the goal.

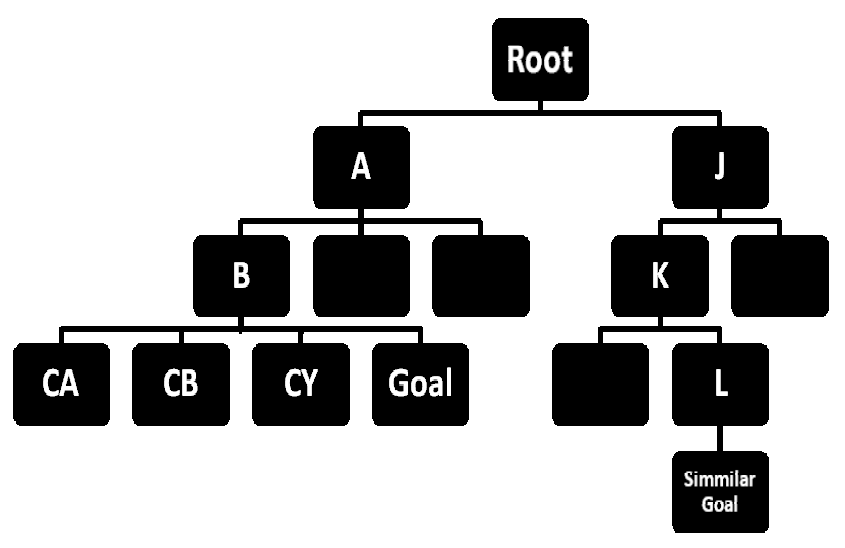

Figure 11 - Information Search Task D1

In one of the difficult information search tasks D1, "find information on council tax arrears", is illustrated in Figure 11. All of the subjects who arrived or went close to obtaining the information went through J, K, L path instead of the A, B path. None of the low literacy subjects were able to obtain the goal, but assumed they had located the information, and therefore abandoned the search early. They seemed totally disoriented while following long trails of links and they seem to keep track of their location but continued to get lost. One of the participants expressed "this seems complicated... too much of reading... not sure where I am currently....". On the other hand, three of six high literacy subjects were able to obtain this goal, while two out of the remaining three came up to J,K and L and abandoned the search assuming they have found the information they were looking for. 
Possibly due to their lack of comprehension of the information they read, the low literacy users often prematurely abandoned their searches, judging that they had reached the goal. We understand this to be of major concern since the incorrect judgement of information as being appropriate might be worse than not being provided with any information at all. This can lead to serious problems such as in the provision of correct but inappropriately interpreted information about health and drug use information due to it being located for the wrong reasons.

\section{CONCLUSION}

Depending on which survey results we use, the occurrence of low literacy in the UK is between 16 to $20 \%$ of the population. We expect that this level of low literacy would be higher in less developed countries. With the increasing use of information technology to provide self-help information, it becomes increasingly important for designers to recognise that interfaces designed primarily for higher literacy users do not work the same way for low literacy users. Understanding the differences between low and high literacy users will help develop new designs that would better support low literacy users find information they need. We should not "dumb down" the interface, i.e. we should not introduce designs that are compatible with the low literacy users because they use simple language or pictographs, but instead, should introduce 'power' features that enable low literacy users to find necessary information. These tools should leverage an understanding of the different ways in which they find and make sense of the information. This will be the focus of future work.

In this study, we highlighted a number of significant differences in the information search and retrieval strategies between high and low literacy participants. Most of the information requires a reading level that prohibits optimal access by low-literacy adults. The low-literacy adult population is a likely audience for Citizens Advice Bureau's information kiosks systems. The size of the population and the identified differences in information search strategies suggests that we should design differently when designing systems for user groups such as those of the CAB. The designs should aim to reduce the user lostness, disorientation and engage the user in a more focused search by reducing the mental effort anticipated by low literacy users.

\section{FUTURE DEVELOPMENT}

It was difficult to draw definitive conclusions from this study due to the small sample size. We intend to carry out further studies that will help us, (i) determine if there is a correlation between levels of literacy, socioeconomic and cultural factors, and how these participants go about finding information, (ii) identify the differences between the search paths that these users take, (iii) analyse the different types of user profiles and information clusters, as these information clusters could comprise several sets of information that are relevant to a user, iv) develop behavioural models of information representation for large and small displays while improving the information search and retrieval strategies for low and high literacy user groups, and (v) develop prototypes to demonstrate the adaptation of the knowledge management technologies such as the Semantic Web and Topic Maps, with designs to support context-based search and retrieval strategies for low and high literacy users. Other future work will identify the factors that will help optimise information systems in an effective manner to help citizens to obtain the information. We need to design technologies that less motivated, low-literate users are able to use to accurately and rapidly obtain the information they need.

\section{ACKNOWLEDGEMENTS}

Our deepest appreciation go to Ms Helen Kelavey from the National Citizens Advice Bureau, Mr Tim Clark, Mr Ken Luxon and Mr Andrew Enever from Barnet Citizens Advice Bureau for giving their fullest support during our study, and for the 12 volunteers in this study for giving so freely of their time. This study has approval from the School's Research Ethics Committee.

\section{REFERENCES}

[1] Aguilar, F. J. (1967). Scanning the Business Environment. New York, NY: Macmillan Co.

[2] Baynham, M. (1995). Literacy Practices UK: Longman

[3] Byström, K. \& Järvelin, K. (1995). Task complexity affects information seeking and use. Information Processing \& Management, 31, (2) (1995). 191-213.

[4] Belkin, N. J. (1980). Anomalous states of knowledge as a basis for information retrieval. Canadian Journal of Information Science, 5, 133-143.

[5] Catledge, L. \& Pitkow, J. (1995). Characterizing browsing strategies in the World Wide Web, Computer Networks and ISDN Systems 27 (1995), pp. 1065-1073.

[6] Campbell, D. J. (1988). Task complexity: A review and analysis. Academy of Management Review, 13, 40-52.

[7] Chlebowska, K. (1990). Literacy for Rural Women in the Third World. Paris: United Nations Educational Scientific and Cultural Organization

[8] Chadwick-Dias, A., McNulty, M., \& Tullis, T. (2003). Web usability and age: How design changes can improve performance. Paper presented at the CUU, Vancouver, BC, Canada.

[9] Kelly, G. A. (1963). A theory of personality: The psychology of personal constructs. New York: Norton.

[10] Kim, Y. M. \& Rieh, S. Y. (2005). Dual-Task Performance as a Measure for Mental Effort in Library Searching and Web Searching. Proceedings of the 68th Annual Meeting of the American Society for Information Science and Technology.

[11] Kuhlthau, C. C. (1991). Inside the search process: Information seeking from the user's perspective. Journal of the American Society for Information Science, 42(5), 361-371.

[12] Longdale, M. \& McCurry, D. (2004). Literacy in the New Millennium Teaching and learning, literacy numeracy in general

[13] Smith, P.A. (1996). Towards a practical measure of hypertext usability, Interacting with Computers $8, \mathrm{pp}$. 365-381.

[14] Stifelman, L. J., Arons, B., Schmandt, C., \& Hulteen, E. A. (1993). VoiceNotes: A Speech Interface for a HandHeld Voice Notetaker. INTERCHI, Amsterdam, April 2429

[15] Summers, K. \& Summers, M. (2005). Reading and Navigational Strategies of Web Users with Lower Literacy Skills

[16] Theofanos, M. F. \& Redish, J. C. (2003). Guidelines for accessible and usable web sites: Observing users who 
work with screen readers, Interactions, X (6), NovemberDecember, 38-51.

[17] Theofan, \& Redish, J. C. (2004). Peak Performance: Redesigning the Portal of the Department of Health and Human Services. User Experience, 3 (6), Spring, 4-7.

[18] Weick, K. E. \& Daft. R.L. (1983). The Effectiveness of Interpretation Systems. In Organizational Effectiveness: A Comparison of Multiple Models, ed. Kim S. Cameron and David A. Whitten, 71-93. New York, NY: Academic Press.

[19] Williams, J., Clemens, S., Oleinikova, K., \& Tarvin, K. (2003). BMRB Social Research for the Department for ducation and Skills (2003). The Skills for Life survey: A national needs and impact survey of literacy, numeracy and ICT skills. London: Department for Education and Skills.

[20] Wong, W., Keith, S. \& Springett, M. (2006). Fit for Purpose Evaluation: The Case of a Public Information Kiosk for the Socially Disadvantaged 